\title{
The mid-infrared spectral characteristics of blazars
}

\author{
Howard A. Smith ${ }^{1}$, F. Massaro ${ }^{2}$, R. D'Abrusco ${ }^{1}$, A. Paggi ${ }^{1}$, \\ P. Cowperthwaite ${ }^{1}$, N. Masetti ${ }^{3}$, M. Giroletti ${ }^{4}$, \\ G. Tosti ${ }^{5,6}$ and M. Landoni ${ }^{7}$ \\ ${ }^{1}$ Harvard-Smithsonian CfA, ${ }^{2}$ Yale, ${ }^{3}$ INAF/IASF, ${ }^{4}$ INAF/IRA, \\ ${ }^{5}$ Perugia Fisica, ${ }^{6}$ Perugia INFN, ${ }^{7}$ INAF/OAB \\ email: hsmith@cfa.harvard.edu
}

\begin{abstract}
We previously reported discovering that blazars have distinctive infrared colors as seen in the WISE mission. Of particular note are $\gamma$-ray blazars, the locus of whose colors roughly overlaps with QSOs contours but which is more tightly delimited and bluer than ULIRGs and Seyferts. Since a large fraction of $\gamma$-ray sources are unassociated with any point source, but are expected to be blazars, identifying a WISE-candidate blazar in the $\gamma$-ray source field enables efficient followup studies. In an effort to better understand why blazars have these colors, we reduced and analyzed Spitzer Infrared Spectrometer (IRS) measurements on 73 blazars of varying types, some at several epochs. These spectra can be used to study the continuum emission (non-thermal), and search for atomic or other spectral features.
\end{abstract}

Keywords. galaxies: active, infrared: galaxies, spectroscopy

\section{Introduction}

The Wide-Field Infrared Survey Explorer (WISE) was an all-sky IR survey with four bands centered near 3.4, 4.6, 12, and $22 \mu \mathrm{m}$ with an angular resolution of about 6 arcsec and $5 \sigma$ point source sensitivities better than $0.08,0.11,1$ and 6 mJy respectively. In our initial paper on the first WISE release (Massaro et al. 2011), and in followup papers on the full sky release (Massaro et al. 2013) we report discovering that blazars have distinctive WISE colors. Figure 1 shows the locus of points for blazars subdivided into categories of BZB, BZQ and mixed (from D'Abrusco et al. 2014).

\section{Spectral characteristics and non-thermal continuum indices}

Two representative IRS spectra are shown in Figure 2, the first from a featureless class with convex shape, and the second from a mixed class with some lines. Other midIR classes are: featureless and concave, concave or convex with broad dust features in absorption or emission, and concave or convex with atomic fine structure lines and/or PAH features. Figure 3 shows the breakdown.

We fit the continua to a two-component power law $-\left(\alpha+\beta \log \left(\nu / \nu_{0}\right)\right)$; Figure 3 shows the distribution.

We acknowledge partial support from NASA grants NNX12AO97G and NNX13AP20G. The work by G. Tosti is supported by the ASI/INAF contract I/005/12/0.

\section{References}

D’Abrusco, R., Massaro, F., Paggi, A., et al. 2014, ApJS, 215, 14

Massaro, F., D’Abrusco, R., Ajello, M., Grindlay, J. E., \& Smith, H. A. 2011, ApJL, 740, L48

Massaro, F., D’Abrusco, R., Paggi, A., et al. 2013, ApJS, 209, 10 


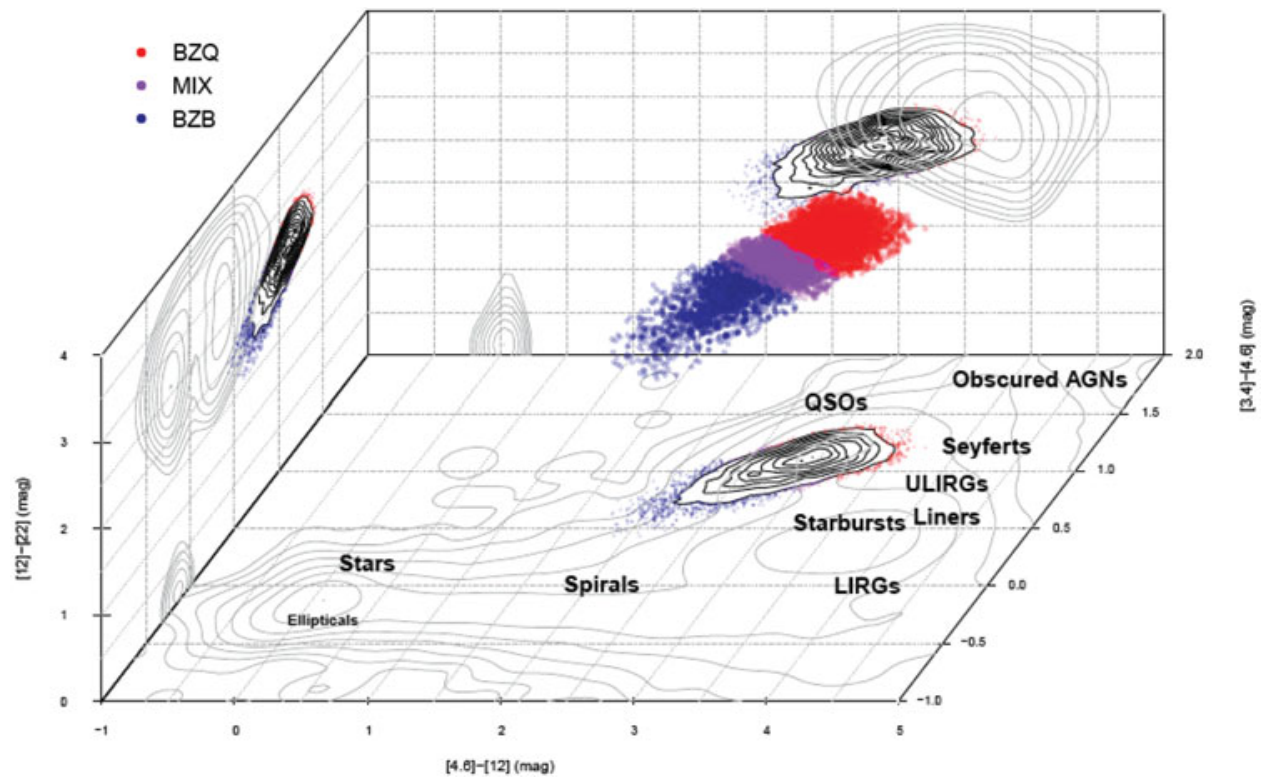

Figure 1. Blazar loci in IR color-color space.
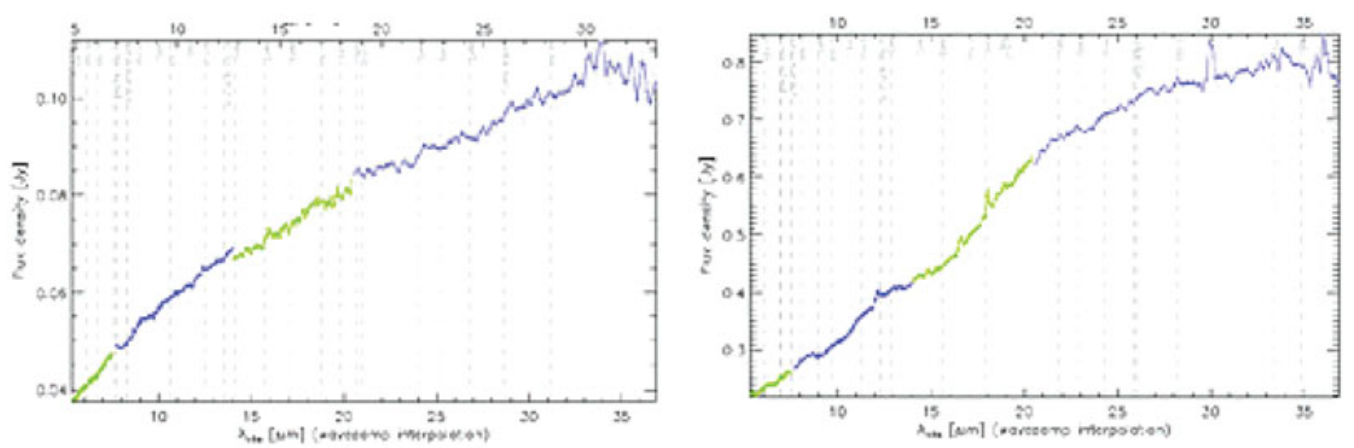

Figure 2. The mid-IR spectra of two blazar classes. Left BZB ON231; right BZQ 2C273.

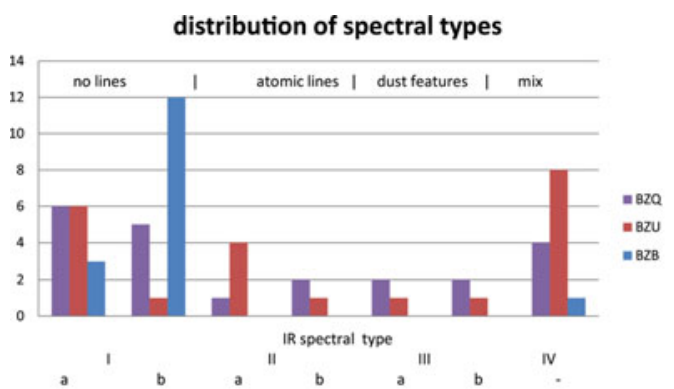

'a': concave continuum shape; 'b': convex shape with $\alpha>1$ with $\alpha<1$

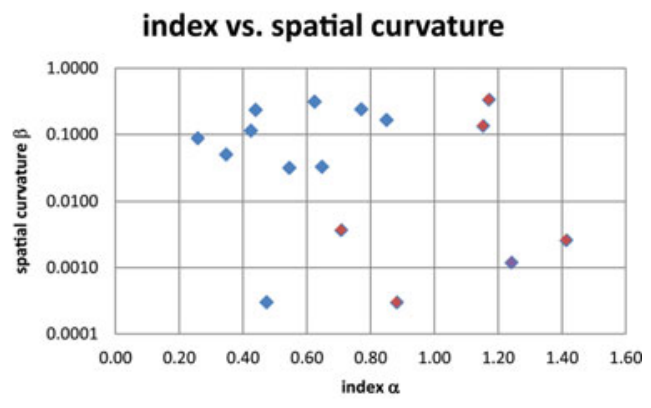

Figure 3. Left: The observed classes of mid-IR spectra in blazars. "a" means concave shape with spectral index $>1$; " $b$ " means convex shape with index $<1$. Right: $\beta$ versus $\alpha$ for blazars. Blue indicates BZB, red are BZU, and violet are BZQ. 